\title{
Blowing the Whistle on Concussion Knowledge and Education in Youth Sport Referees
}

\section{Colin King (D) \\ Erin Coughlan}

School of Kinesiology, Acadia University, Wolfville, NS, Canada
Correspondence: Colin King

School of Kinesiology, Acadia University,

550 Main Street, Wolfville, NS, B4P 2R6,

Canada

Tel + I 902585 I309

Email colin.king@acadiau.ca
Purpose: To gain an understanding of referee concussion knowledge, what sporting organizations were doing to prepare their referees to recognize concussions in youth sport, and what role referees could play in overall concussion safety.

Materials and Methods: A total of 134 referees participated in the study across four provincial sporting organizations (rugby, soccer, ice hockey, and football). Participants completed a concussion knowledge questionnaire that explored knowledge about concussion education, recognition, management, preparedness, and the role of the referee in overall concussion management.

Results: Overall, the sample was found to be moderately knowledgeable about concussions. Referees that received previous formal concussion education had statistically significant higher average scores when compared to those without formalized concussion education $(P=0.001)$. However, only $24 \%$ of respondents reported receiving any formal education from their sporting body or referee organization. About $85 \%$ felt that referees could play an important role in concussion recognition in youth sport, but only $41 \%$ felt they were adequately equipped with the knowledge and skills to recognize these injuries.

Conclusion: The findings from this study suggest that national, provincial, and individual sporting organizations should ensure that all stakeholders (including referees) are formally educated about concussions to encourage a safety-first environment for youth athletes. Educational sessions should focus on the general aspects of SRC while also focusing on the specific role of the referee in concussion recognition.

Keywords: sports-related concussions, officials, injury prevention, injury recognition

\section{Introduction}

Recently, interest in sports-related concussions (SRC) has grown immensely in sports and scientific communities alike, in order to better understand this complex injury and its impact on athletes' lives. ${ }^{1}$ Researchers have frequently explored concussion knowledge in many different stakeholder groups, including: athletes, ${ }^{2}$ coaches, ${ }^{3}$ parents, ${ }^{4}$ and medical professionals. ${ }^{5}$ However, referees are a group who are often misrepresented in the previously published literature. This is concerning as referees have the potential to mitigate the risk of further injury following an SRC, especially in the absence of qualified medical professionals (which occurs during many youth sporting events). ${ }^{6}$ Given these gaps in the literature, the purpose of this study was to explore SRC knowledge in a sample of referees from various collision and contact sports. Additionally, we explored how referees were educated about concussions, what sporting organizations were doing to prepare their referees to recognize concussions in youth sport, and what role could referees play in overall SRC safety. 


\section{The Role of the Referee in Sport}

Referees have been historically understudied in sport, with a small body of literature focusing on constructs such as the stresses associated with the role as a referee, the physiological and psychological demands of the job, and the impact of biases and influences on decision-making. ${ }^{7}$ The specific roles of a referee vary greatly, as the level of interaction with athletes and their influence on the outcome of a game is unique to each individual sport. ${ }^{8}$ Of particular relevance to youth sport are interactor referees, as described by Farrow et $\mathrm{al}^{8}$ as referees that monitor a multitude of rules and cues while constantly interacting with athletes and moving with the play. To be considered an effective interactor referee (such as in soccer or ice hockey), one must possess a number of characteristics including strong communication skills and quick decisionmaking abilities, while integrating these with expert-level knowledge of the rules of the game. ${ }^{9}$ These referees must also be able to carefully and critically observe play while simultaneously following it, requiring oftentimes intense physical exertion and constant shifting of their focus. ${ }^{7}$

At the foundation of refereeing lies two key responsibilities: upholding the integrity of the sport by enforcing the laws and rules of the game and ensuring player safety. ${ }^{10}$ While one could argue that a referee protects player safety by simply enforcing the rules of the sport, the referee's role in player safety is in reality more complex and multifaceted. ${ }^{11}$ Referees can punish illegal or dangerous behavior by awarding a penalty to the offending team, but as an interactor they are also tasked with identifying potential serious injuries or threats to players safety. ${ }^{11}$

\section{The Potential Role of the Referee in Concussion Safety}

In collision and contact sports, interactor referees have the power to stop the game if there is concern for player safety, namely a potential injury. This becomes vitally important in the initial management of SRCs, as these injuries require immediate recognition and removal from play, to ensure proper assessment, treatment, and prevention of catastrophic conditions such as second impact syndrome. ${ }^{12}$ Referees could potentially act as another moderator of concussion safety by recognizing potential SRCs and stopping play to allow for proper medical assessment and management by qualified individuals. ${ }^{13}$ However, most research in injury prevention and management of SRCs has largely been directed towards athletes, coaches, and parents, not referees. ${ }^{14,15}$
The Rugby Union is one organization that is forward thinking about the referee's role in concussion safety, with the development of their Blue Card Programme. ${ }^{16}$ This program educates referees about concussion recognition and then empowers referees with the ability to remove players from the field if a SRC is suspected. Once removed, a player must then have an assessment from a qualified medical professional, followed by a standardized RTP protocol before allowing to be returned to the pitch. Initial findings from a study conducted on a trial of this Blue Card Programme found that $95 \%$ of referees surveyed felt that they had sufficient training for this new responsibility through the formalized concussion education program. ${ }^{16}$

\section{Referee Concussion Knowledge}

As previously mentioned, concussion recognition and management knowledge has remained largely unexplored in referees, with only a few studies being dedicated to this stakeholder group. One such study investigated SRC knowledge in amateur rugby coaches and referees. ${ }^{13}$ These researchers found that referees and coaches had an overall understanding of general SRC signs and symptoms but struggled in areas such as objectively recognizing a SRC and understanding specific management protocols. Additionally, referees who reported previous formalized concussion education were stronger in these two areas when compared to participants without formal education about concussions.

Gallagher et $\mathrm{al}^{17}$ investigated the SRC knowledge of professional and amateur rugby referees. This study found that $72 \%$ of respondents felt they could subjectively diagnose an SRC based on knowing an athlete's reported symptoms but were not as confident in recognizing potential concussions based on objective signs. These researchers also found that only $45 \%$ had received previous formalized concussion education. Additionally, Kroshus et $\mathrm{al}^{18}$ found that officials with more formalized concussion knowledge demonstrated greater self-efficacy when calling injury timeouts, therefore fostering an environment that supported overall SRC safety.

These previous studies all focused on SRC knowledge of referees from professional and college/university sports. No known studies have focused on youth sport, which is of grave concern because: youth populations are more susceptible to SRCs, youth sport referees are often less experienced, and medical professionals are not present at each and every game. ${ }^{6,12}$ Based on these gaps in the literature, we decided to explore SRC knowledge in 
a sample of youth sport referees from various collision and contact sports.

\section{Materials and Methods Study Design}

This cross-sectional study used an online questionnaire to collect data from youth sport referees. Purposive sampling was used to target Nova Scotia referees who held a referee certification in either ice hockey, soccer, football, or rugby, and had experience with refereeing youth sport under the age of 18 . Ethical approval was obtained by the Acadia University Research Ethics Board before the study commenced.

\section{Study Setting and Recruitment}

Four provincial sporting organizations in Nova Scotia, Canada were selected for this study: ice hockey, soccer, football, and rugby. Each organization was contacted by one of the researcher's who described the study objectives, inclusion criteria for participation, and expectations for participants. Once confirmed, each sporting organization emailed the study information and link to the online questionnaire to all eligible referees within their organization. Informed written consent was obtained from all participants prior to completing the questionnaire, in accordance with the Declaration of Helsinki. If a participant was under the age of 18 , additional written consent was also obtained from a parent or guardian before they were eligible to complete the questionnaire. The first page of the online questionnaire acted as the informed written consent by outlining the purpose of the study and identifying the specific terms of participation. By following the online cues, each participant provided consent. All questionnaires were completed anonymously, so responses could not be identified. Therefore, participants could not withdraw their responses once the questionnaire was submitted.

\section{Participants}

A total of 134 referees participated in the study from the four provincial sporting organizations. However, 35 responses were considered incomplete and were therefore removed from the study sample. Therefore, a total of 98 referees ( $74 \%$ male; $62 \%$ 35 years of age and older) were used for the data analysis. $74 \%$ of the referees had more than 6 years of refereeing experience, and each sporting organization was represented in the sample ( soccer $=53 \%$; ice hockey $=18 \%$; football $=15 \%$; rugby $=$ $13 \%)$. The demographic characteristics of the sample are presented in Table 1.
Table I Demographic Characteristics of Participants ( $n=98)$

\begin{tabular}{|l|l|}
\hline & Frequency (\% of Total Sample) \\
\hline Sex & $73(74.4)$ \\
Male & $24(24.4)$ \\
Demale Not Wish to Disclose & $1(1.2)$ \\
\hline Age & \\
Under 18 & $2(2.1)$ \\
19-24 & $19(19.4)$ \\
$25-34$ & $15(15.3)$ \\
$35-49$ & $31(31.6)$ \\
$50-64$ & $22(22.4)$ \\
$65+$ & $9(9.2)$ \\
\hline Experience & \\
$0-2$ years & $6(6.1)$ \\
$3-5$ years & $20(20.4)$ \\
$6-10$ years & $27(27.5)$ \\
II+ & $45(46.0)$ \\
\hline Sport & \\
Rugby & $13(13.3)$ \\
Hockey & $18(18.4)$ \\
Soccer & $52(53.0)$ \\
Football & $15(15.3)$ \\
\hline
\end{tabular}

\section{Concussion Knowledge Questionnaire}

The online questionnaire was modeled from a previous concussion knowledge survey ${ }^{19}$ and modified to reflect the most recent Consensus Statement on Concussion in Sport. ${ }^{12}$ The questionnaire consisted of 27 questions that took approximately 15 minutes to complete and was hosted on a secured internal survey system from the primary researcher's home institution. All responses were submitted anonymously. Questions covered the following topics: 1) demographics and sport experience; 2) general SRC knowledge; 3) recognition of SRC signs and symptoms (including distractor symptoms); 4) general attitudes towards SRC and the role(s) of referee in management); and 5) perceived confidence in recognizing potential SRCs. Questions included a mixture of dichotomous, Likert scale, and open-ended questions. The questionnaire was reviewed for face validity by three content experts (all certified athletic therapists who were also familiar with questionnaire development) before the questionnaire was sent to participants.

\section{Data Analysis}

All completed data from the questionnaires were entered into Microsoft Excel 2016 (Redmond, WA) for further analysis. 
Table 2 General Sports-Related Concussion Knowledge

\begin{tabular}{|c|c|}
\hline Statement (Correct Response) & $\begin{array}{c}\text { Frequency } \\
\text { with Correct } \\
\text { Response (\% } \\
\text { of Total } \\
\text { Sample) }\end{array}$ \\
\hline Symptoms of a concussion will always appear immediately following following the impact. (FALSE) & $93(95)$ \\
\hline There are no risks associated with returning to a game while still concussed if it is only a mild one. (FALSE) & $93(95)$ \\
\hline People who have had one concussion are more likely to have another concussion. (TRUE) & $82(84)$ \\
\hline Protective equipment such as a helmet/scrum hat/head guard can prevent someone from receiving a concussion. (FALSE) & $51(52)$ \\
\hline In order to be diagnosed with a concussion, you have to be knocked out. (FALSE) & $96(98)$ \\
\hline A concussion can only occur if there is a direct hit to the head. (FALSE) & $91(93)$ \\
\hline Children usually take longer to recover from concussions than adults. (TRUE) & $34(35)$ \\
\hline If a player is knocked unconscious they should be transported to the hospital immediately. (TRUE) & $77(79)$ \\
\hline Sometimes a second concussion can help a person remember things that were forgotten after the first concussion. (FALSE) & $90(92)$ \\
\hline A concussion must be diagnosed by a certified medical professional. (TRUE) & $73(74)$ \\
\hline $\begin{array}{l}\text { After a concussion occurs, brain imaging (eg, CAT Scan, MRI, X-Ray, etc.) typically shows visible physical damage (eg, bruise, } \\
\text { blood clot) to the brain. (FALSE) }\end{array}$ & $51(52)$ \\
\hline A player may have a concussion without the presence of visible symptoms. (TRUE) & $95(97)$ \\
\hline After 10 days, symptoms of a concussion are usually gone. (TRUE) & $22(22)$ \\
\hline The current treatment for concussion is complete brain rest. (FALSE) & $30(31)$ \\
\hline Concussions can sometimes lead to emotional disruptions. (TRUE) & $94(96)$ \\
\hline A player with a suspected concussion should be immediately removed from play. (TRUE) & $97(99)$ \\
\hline There is rarely a risk to long-term health and well-being from multiple concussions. (FALSE) & $83(85)$ \\
\hline
\end{tabular}

Data was also analyzed using the Statistical Programme for Social Sciences (SPSS) (IBM SPSS, Chicago), version 24. Descriptive statistics (total number of responses, means, standard deviations, percentages) were established to provide an overall summary of the data. Participants were provided with a "general SRC knowledge score" (out of a total possible score of 18) based on the correctness of their answers. Participants were also provided with a "recognition of common concussion signs and symptoms" score out of a possible 16. Further statistical analysis was also completed to explore significant differences in SRC knowledge. Independent $\mathrm{t}$-tests were used to derive significant differences in knowledge based on age, years of experience, history of previous concussion, and previous formalized SRC education $(P<0.05)$. Single Factor ANOVAs were also used to compare knowledge amongst referees from different sporting organizations (football, hockey, rugby, hockey) $(P<0.05)$.

\section{Results}

\section{General Concussion Knowledge}

To gauge general SRC knowledge in the sample, participants were asked to determine whether a variety of statements surrounding SRC knowledge were true or false. These statements placed an emphasis on common concussion myths and SRC information deemed to be most pertinent to referees (SRC recognition and initial management). The findings from these questions are provided in Table 2. Out of a possible score of 18, participants averaged $12.97( \pm 1.74)$ correct responses. Additionally, there were no significant differences found between age, years of experience as a referee, sports, or between those referees with or without a history of concussion. However, those with previous formal concussion education had statistically significant higher average scores when compared 
to those without history of formal education $(13.75 \pm 1.29$ vs $12.67 \pm 1.63 ; P=0.001)$. Encouragingly, participants recognized that there are associated risks with returning to a game with a concussion and recognized the importance of removing a player with a suspected concussion immediately from play. However, some questions proved to be more challenging for referees. Only $35 \%$ of the sample recognized that children usually take longer to recover from a concussion than adults. In addition, $68 \%$ of the referees also incorrectly indicated that the current best recommended treatment for a concussion is complete cognitive/physical rest until asymptomatic.

\section{Signs/Symptoms Recognition}

In this section, we provided participants with a list of possible SRC signs/symptoms and asked them to determine which were possible signs/symptoms, and which were distractors (see Figure 1). As seen in the figure, participants appeared to understand the most commonly reported SRC signs/symptoms (eg headache, trouble balancing, dizziness), however had more difficulty identifying distractor symptoms. The average number of correct responses was $13.18( \pm 2.15)$ out of a possible 16, with no significant differences arising between age, experience level, or sports. Participants who reported a history of personal concussion had significantly higher recognition scores than those without (13.75 \pm 1.61 vs $12.17 \pm 3.46$; $P=0.070$ ). Additionally, those with formal concussion education also scored higher than those with no formal education $(13.75 \pm 1.77$ vs $13.08 \pm 2.56 ; P=0.070)$.

\section{Sources of Concussion Knowledge}

Participants' origins of concussion knowledge were diverse and often stemmed from multiple areas from their professional and personal lives. Most referees felt that their concussion knowledge came from personal sporting experiences (79\% of respondents), experiences as a referee $(70 \%)$, and/or history with their own concussion $(47 \%)$. Of concern, only $24 \%$ of the sample reported receiving any formal SRC education from their sporting body or referee organization. When responses were further broken down by sport, rugby referees had the highest rate of formal concussion education (87\% of rugby referee respondents) compared to all other sports. All rugby referees also reported receiving this education by their governing sport organization. The formal SRC education rates for other sports included: soccer $(31 \%)$, hockey $(41 \%)$, and football $(56 \%)$.
When asked about current SRC education practices for the referee population, $87 \%$ felt that more could be done to educate youth sport referees about concussion recognition and management. Participants were asked to further explain their answer (through a text-based open-ended answer), and it was clear that many felt that mandatory SRC education should be included as part of all referees' training. Box 1 shows representative quotations from these text-based responses.

\section{The Potential Role of the Referee in Concussion Safety}

Referees from the sample felt that multiple stakeholders could potentially play an important role in SRC recognition and management in youth sport, instead of placing the sole responsibility on a coach. Most importantly, $85 \%$ of referees agreed or strongly agreed that referees can in fact

Box I Representative Quotations from the Question "Do You Believe More Could Be Done to Educate Youth Sport Referees in Concussion Recognition and Initial Management?"

I. "In the training of youth sport officials, there should be a section on concussion recognition and initial management (ie when to call an injury timeout) similar to the specific information supplied during coach's training."

2. "Everyone should know how to spot a concussion and what to do. Referees should be empowered to call medical timeouts. However coaches and athletes need to be on board with the seriousness and severity. The culture in sport needs to shift such that medical timeouts for head injuries becomes more tolerated and normalized." 3. "Education is always important. People need to be frequently reminded of the need to stop play at any chance of a head injury. Knowing the possible effects of ignoring the signs is valuable, people need to be sensitive to possible outcomes."

4. "There is currently no formal concussion education training in my association. Many have indicated that having the education may open issues of liability in the case of players who continue to participate after a suspected concussion. I believe that without proper education and support from our provincial/governing body, athletes may be at risk."

5. "At the higher-level games, the referees have more confidence to be stopping games for potential head injuries, but it is the youth games where they sometimes lack the knowledge/recognition.

Every year, as a referee, you have to take a refresher course at the beginning of the season so it would be a good opportunity to add some concussion recognition material in there in case there is a situation where they have to make a decision."

6. "It is definitely the referees at the youth level who need more help with it because there is usually limited coaching staff, and not always a full team of officials. And generally there is a lack of knowledge and experience among people at that level, both referees and athletes." 


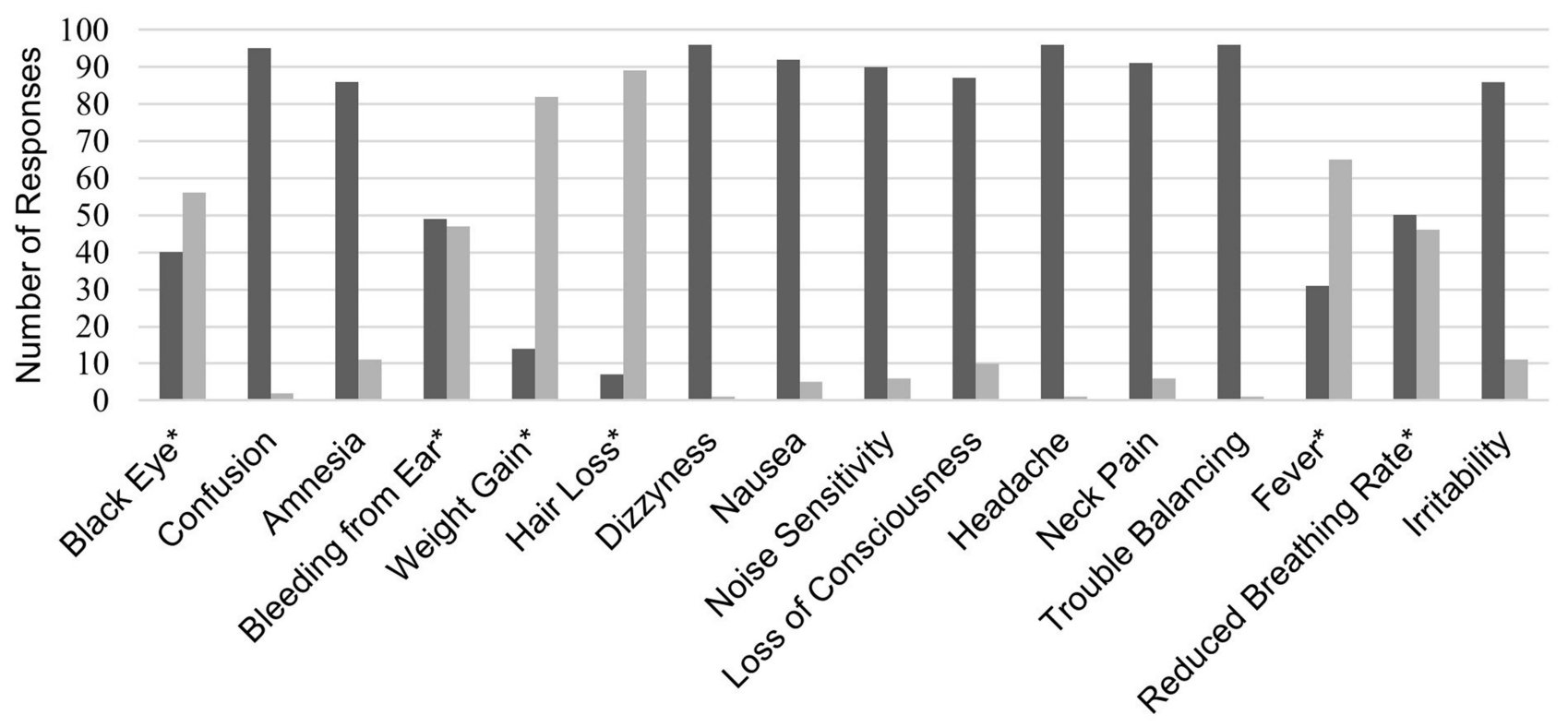

Figure I Dark Gray indicated it was selected as a sign or symptom of a concussion. Light Gray indicated it was selected as a distractor sign or symptom. *Distractor sign or symptom of a concussion.

play an important role in concussion recognition. However, when asked whether they felt equipped to aid in SRC recognition by stopping play for a medical timeout, just $41 \%$ of participants responded with yes. Furthermore, those who had received formal education felt more confident to recognize a potential concussion compared to those who did not (62\% vs $27 \%)$.

\section{Discussion}

The purpose of this study was to explore referee SRC knowledge, sources of SRC knowledge, what sporting organizations were doing to prepare their referees to recognize concussions in youth sport, and what role could referees play in overall SRC safety. Two main themes emerged from the findings: 1) the need for formalized concussion education for youth sport referees, and 2) identifying the potential role for referees in overall concussion safety.

\section{Formalized Concussion Education in Referees}

A key finding from our study was that only $24 \%$ of participants received formal concussion education from their national, provincial, or individual sporting organization. Additionally, referees who had received formal SRC education demonstrated significantly $(P=0.001)$ higher average SRC knowledge scores than those who had not. Signs and symptoms scores were also higher for those with formal SRC education, the difference approaching but not reaching statistical significance $(\mathrm{P}=0.070)$. Griffin et $\mathrm{al}^{13}$ found similar results in amateur rugby referees where previous concussion education led to higher scores in sign/symptom recognition, understanding consequences of SRC, and knowledge of return-to-play protocols.

While SRC education will not ensure that recognition or proper management is always adhered to, it is an important step towards increasing SRC awareness and knowledge. ${ }^{20}$ To increase the effectiveness of SRC education, many studies have recommended a more detailed consideration of knowledge translation principles to increase the likelihood of knowledge retention and demonstration of positive behaviors. ${ }^{15}$ Research has also shown that multimodal approaches (using multiple resources) and tailoring the content specifically to the intended audience, leads to increased knowledge compared to using singular, generalized resources. ${ }^{15}$

In our study, rugby referees were found to have the highest average general SRC knowledge and sign/symptom scores. Upon further investigation of this trend, it was found that rugby is one of the few sports in Canada that has mandatory SRC education now in place for their referees. ${ }^{21}$ Two online courses must be completed annually, addressing topics such as: SRC recognition, concussion management, return-to-play protocols, as well as the role of the rugby referee in SRC safety. The effectiveness of this program has not been investigated; however, one could speculate that this education is effective based on the trends found in similar studies (including our study). Further research is warranted to consider sporting 
cultures and diverse educational strategies that may influence SRC knowledge and specific changes in behavior.

Education is an important aspect of SRC safety, however we understand that education alone does not always lead directly to behavior change. ${ }^{22}$ Exploring SRC behaviors in referees is outside the scope of this study but future research will need to investigate this multifaceted construct. As an example, a study by Kroshus et al ${ }^{18}$ found that those referees with more SRC knowledge also demonstrated greater self-efficacy, a key predictor in behavior change. These researchers posited that those with higher levels of SRC knowledge also had increased confidence in their ability to call an injury time-out during challenging game scenarios. Therefore, future research should continue to explore how to best enhance referee self-efficacy in SRC prevention, recognition, and management.

\section{The Potential Role of the Referee in Concussion Safety}

The role of the referee is challenging and dynamic as they are charged with making quick decisions (using proper judgement and interpretation of the laws of the game), maintaining clear communication with athletes, coaches and other referees, protecting player welfare, changing field position to best view play, and maintaining the integrity of their sport. ${ }^{7}$ The role of the referee in overall sport safety has been traditionally confined to simply enforcing the rules of the game. ${ }^{23}$ However, some researchers have advocated for an increased role for referees in sport safety, especially in the recognition and prevention of SRCs. Clacy et $\mathrm{al}^{23}$ investigated the perceived responsibilities of rugby union stakeholders in the prevention of SRCs. These researchers found that there were significant discrepancies in how referees understood their role-specific responsibilities in concussion prevention. Additionally, the majority of referees in this sample did not feel that they were directly a part of SRC safety. Similar findings were found in our study as $85 \%$ of referees felt that they could play a more significant role in SRC safety. While enforcing the rules can minimize dangerous behaviors and aid in the prevention of common SRC mechanisms, referees can also play a vital role in protecting athletes by recognizing initial signs of a SRC (eg recognizing balance/gait difficulties following a contact mechanism) and removing athletes from play to be properly medically evaluated. ${ }^{13,18}$ This becomes even more important in youth sport, where there is often no medical staff present to initiate this removal from play. ${ }^{6}$ If referees were more knowledgeable about SRC and had a clearly defined role (eg the Blue Card program), then they could potentially act as another important stakeholder in SRC recognition/prevention. This can even remove pressures from the coach/athlete dynamic when coaches are responsible for SRC recognition in the absence of qualified medical professionals. $^{6}$

\section{Future Directions}

The findings from this study, supported by previously published literature, demonstrate that with the proper support and education from their sporting organizations, youth sport referees may be able to assist in recognizing potential SRCs and mitigate risks of further injury in young athletes. Education that focuses on the specific role of the referee in concussion recognition and management may help referees to feel more confident to stop play for proper medical assessment by a qualified health professional. Educational approaches should use a multimodal approach with various types of resources to encourage engagement and retention within this population. Additional support from stakeholders including athletes, coaches, health professionals, and parents could also help to foster an overall sport culture that prioritizes SRC safety. Empowering referees to recognize SRCs, and in turn mitigate additional harm following SRC, is important for national, provincial, and individual sporting organizations to consider, particularly in youth sport settings where qualified health professionals are often absent. ${ }^{6}$

Future research should continue to explore the role of the referee in SRC safety, as literature in the area is extremely limited. Perceptions of youth sport stakeholders in SRC safety should be further investigated to identify how to create a supportive environment for referees. Future research should also investigate specific educational formats and strategies that effectively target referees to increase their SRC knowledge and self-efficacy to make positive decisions.

\section{Limitations}

Some limitations should be acknowledged upon the completion of this study. Few participants under the age of 18 completed this study, potentially due to the lengthy consent process that was required. Since these individuals are often less experienced referees, future research should attempt to investigate concussion knowledge of less experienced referees in youth sport settings. Additionally, participants in this study were self selected (by responding to the initial email recruitment email that was sent out by the referee organization) so there is the potential for a response bias, as those with a personal interest in SRCs may have been more likely to complete this study. 
Finally, the questionnaire was completed by participants online, without a defined time limit, therefore participants could have searched for information to help them answer the SRC knowledge questions.

\section{Conclusion}

The purpose of this study was to explore SRC knowledge in a sample of referees from various collision and contact sports. Additionally, we explored how referees were educated about concussions, what sporting organizations were doing to prepare their referees to recognize concussions in youth sport, and what the potential role for referees is in overall concussion safety. Referees were found to be moderately knowledgeable about SRCs, but many felt that they were not equipped to recognize a potential SRC in their sport. Most referees did not receive formalized SRC education from their sporting organization and felt that more could be done to educate youth sport referees about SRC, while focusing on the specific role of the referee in concussion recognition. These findings suggest that national, provincial, and individual sporting organizations should ensure that all stakeholders (including referees) are formally educated about SRCs to encourage a safety-first environment for youth athletes. Organizations could look to Rugby Canada ${ }^{21}$ and the Blue Card Programme ${ }^{16}$ as practical examples of how to create effective concussion education policies and practices, while ensuring that any program is specifically designed for referees in a particular sport. Established knowledge translation frameworks can also be implemented to help improve referee knowledge about SRC's, while also developing the skills required to make competent decisions in recognizing a potential head injury. ${ }^{24}$

\section{Acknowledgments}

The authors acknowledge the support of the Nova Scotia provincial sport organizations for soccer, ice hockey, rugby, and football and to the referees who volunteered to participate. Funding for this project was provided by the Acadia University Honours Summer Research Grant Program.

\section{Disclosure}

The authors report no conflict of interest or disclosure of financial interest.

\section{References}

1. Chancellor SE, Franz ES, Minaeva OV, et al. Pathophysiology of concussion. Semin Pediatr Neurol. 2019;30:14-25. doi:10.1016/j. spen.2019.03.004
2. Register-Mihalik JK, McLeod TCV, Linnan LA, et al. Relationship between concussion history and concussion knowledge, attitudes, and disclosure behavior in high school athletes. Clin J Sport Med. 2017;27(3):321-324. doi:10.1097/JSM.0000000000000349

3. Kay MC, Williams RM, McLeod TCV, et al. Concussion knowledge, attitudes, and perceived level of involvement by American youth sport coaches in concussion management. Br J Sports Med. 2017;51(11):A63-A63. doi:10.1136/bjsports-2016-097270.163

4. Rice T, Curtis R. Parental knowledge of concussion: evaluation of the CDC's "Heads up to parents" educational initiative. J Safety Res. 2019;69:85-93. doi:10.1016/j.jsr.2019.02.007

5. Salisbury D, Kolessar M, Callender L, et al. Concussion knowledge among rehabilitation staff. Bayl Univ Med Cent. 2017;30(1):33-37.

6. Russel K, Ellis MJ, Bauman S, et al. Legislation for youth sport concussion in Canada: review, conceptual framework, and recommendations. Can J Neurol Sci. 2017;44(3):225-234. doi:10.1017/cjn.2016.423

7. Hancock DJ, Rix-Lievre G, Cote J. Citation network analysis of research on sport officials: a lack on interconnectivity. Int Rev Sport Exerc Psychol. 2015;8(1):95-105. doi:10.1080/1750984X.2015.1022202

8. Farrow D, Baker J, MacMahon C. Developing Sport Expertise. 2nd ed. New York, NY: Routledge; 2013.

9. Guillen F, Feltz DL. A conceptual model of referee efficacy. Front Psychol. 2011;2:1-5. doi:10.3389/fpsyg.2011.00025

10. Isidori E, Muller A, Kaya S. The referee as educator: hermeneutical and pedagogical perspectives. Phys Cult Sport Stud Res. 2012;56 (1):5-11. doi:10.2478/v10141-012-0023-2

11. Mascarenhas DRD, Collins D, Mortimer P. Elite refereeing performance: developing a model for sport science support. Sport Psychol. 2005;19(4):364-379. doi:10.1123/tsp.19.4.364

12. McCrory P, Meeuwisse W, Dvorak J, et al. Consensus statement on concussion in sport - the 5 th international conference on concussion in sport. Br J Sports Med. 2017;51(11):838-847.

13. Griffin SA, Ranson C, Moore I, et al. Concussion knowledge and experience among Welsh amateur rugby union coaches and referees. BMJ Open Sport Exerc Med. 2017;3(1):1-7. doi:10.1136/bmjsem-2016-000174

14. Chrisman SP, Schiff MA, Chung SK, et al. Implementation of concussion legislation and extent of concussion education for athletes, parents, and coaches in Washington State. Am J Sport Med. 2014;42 (5):1190-1196. doi:10.1177/0363546513519073

15. Cusimano MD, Zhang S, Topolovec-Vranic J, et al. Pros and cons of 19 sport-related concussion educational resources in Canada: avenues for better care and prevention. Front Neurol. 2018;9:872. doi:10.3389/fneur.2018.00872

16. Sullivan J, Kyal-Grey A, Handcock P. Blue card: referees' perspectives of a rugby union concussion recognition and management programme. Br J Sports Med. 2017;51(11):A80.

17. Gallagher T, Mias E, Kipps C. Recognition and knowledge of on-field management of concussion amongst English professional, semi-professional and amateur rugby union referees. Br J Sports Med. 2017;51(11):A82. doi:10.1136/bjsports-2016-097270.212

18. Kroshus E, Parsons J, Hainline B. Calling injury timeouts for the medical evaluation of concussion: determinants of collegiate football officials' behavior. $J$ Athl Train. 2017;52(11):1041-1047. doi: $10.4085 / 1062-6050-52.11 .17$

19. Rosenbaum AM, Arnett PA. The development of a survey to examine knowledge about and attitudes toward concussion in high-school students. J Clin Exp Neuropsychol. 2010;32(1):44-55. doi:10.1080/ 13803390902806535

20. Caron JG, Rathwell S, Delaney JS, et al. Development, implementation and assessment of a concussion education programme for high school student-athletes. J Sports Sci. 2018;36(1):48-55. doi:10.1080/ 02640414.2017.1280180

21. Rugby Canada's PlaySmart: putting players first. Rugby.ca; 2016. Available from: https://rugby.ca/uploads/Documents/Rugby Canada_s_PlaySmart_-_Putting_Players_First.pdf. Accessed February 12, 2020. 
22. Mrazik M, Dennison CR, Brooks BL, et al. A qualitative review of sports concussion education: prime time for evidence-based knowledge translation. Br J Sports Med. 2015;49(24):1548. doi:10.1136/ bjsports-2015-094848

23. Clacy A, Sharman R, Goode N, et al. Responsibilities in the prevention of concussion in community rugby union. Procedia Manuf. 2015;3:1173-1180. doi:10.1016/j.promfg.2015.07.195
24. Guo D, Verweel L, Reed N. Exploring gaps in concussion knowledge and knowledge translation among coaches of youth female hockey. Clin J Sport Med. 2019;29(6):486-493.

\section{Publish your work in this journal}

Open Access Journal of Sports Medicine is an international, peerreviewed, open access journal publishing original research, reports, reviews and commentaries on all areas of sports medicine. The manuscript management system is completely online and includes a very quick and fair peer-review system. Visit http://www.dovepress. com/testimonials.php to read real quotes from published authors. 